\title{
MAY O 41990
}

R. V. F. Janssens ${ }^{a}$, M. P. Carpenter ${ }^{a}$, M. W. Drigert ${ }^{b}$, P. B. Fernandez ${ }^{a}$, E. F. Moorea, D. Ye ${ }^{c}$, I. Ahmada, K. B. Beardc, I. G. Beardend, Ph. Benetd, P. J. Dalyd, U. Gargc, 2. W. Grabowskid, T. L. Khoo a, W. Reviolc and

F. L. H. Wolfs ${ }^{a}$

a: Argonne National Laboratory, Argonne, IL 60439, USA

b: Idaho National Engineering Laboratory, Idaho Falls, ID 83415, USA

c: University of Notre Dame, Notre Dame, IN 46556, USA

CONF-900450--2

d: Purdue University, West Lafayette, IN 47907, USA

DE9 010103

\section{INTRODUCTION}

First experimental evidence for a new region of superdeformation near An190 is only about a year old: a band of 12 transitions with an average energy spacing of $37 \mathrm{keV}$, an average dynamic moment of inertia $\mathrm{J}(2)$ of 110 $\hbar^{2} \mathrm{MeV}^{-1}$ and an average quadrupole moment of $18 \pm 3 \mathrm{eb}$ was observed in $19 \mathrm{I}_{\mathrm{Hg}}$ (Ref. 1). This result confirmed predictions ${ }^{2-5}$ made using a variety of theoretical approaches for the occurrence of superdeformed nuclei in this region with a major to minor axis ratio of 1.65:1 ( $\left.\beta^{\sim 0.5}\right)$. Several important questions were raised by the original experimental result. First, the dynamic moment of inertia $J^{(2)}$ was found to increase steadily with rotational frequency hw. Mean-field calculations which attempt to reproduce variation in $J$ (2) have suggested that such a rise may be attributed to three major factors which couid contribute separately or cooperatively: (i) shape changes as a function of hw (centrifugal stretching), (ii) changes in pairing at large deformations ${ }^{6}$ and (iii) occupation of specific high-j orbitals 6,7 (i $13 / 2$ protons and $j_{15 / 2}$ neutrons). Second, in the work of Ref. 1 the superdeformed (SD) band was found to decay only to the $17 / 2^{+}$state and it was neither possible to obtain a firm indication of the spins of the superdeformed states nor to assess whether the link between SD states and the yrast levels is statistical in nature as in the A 150 region $^{8}$ or occurs only through specific. transitions. Finally, questions regarding the existence of ather SD nuclei in this region and the limits in $N$ and 2 of the SD regions also needed attention.

In the past year, an impressive number of new results has become available; some of these will be reviewed in this contribution and in the following one by F.S. Stephens. Here, we shall first summarize the present experimental situation conceming ${ }^{192} \mathrm{Hg}$, the nucleus regarded as the analog 8 
of ${ }^{152}$ Dy for this SD region in that shell gaps are calculated 5 to occur at large deformation for $\mathrm{Z}=80$ and $\mathrm{N}=112$. Proton and neutron excitations out of the ${ }^{192} \mathrm{Hg}$ core will then be reviewed with particular emphasis on ${ }^{191} \mathrm{Hg}$ and 193Tl. The presentation will conclude with a brief tiscussion on limits of the SD region for neutron deficient Hg nuclei.

2. SUPERDEFORMATION IN $192 \mathrm{Hg}$

The SD band of ${ }^{192} \mathrm{Hg}$ as measured at ATLAS with the ${ }^{160} \mathrm{Gd}\left({ }^{36} \mathrm{~S}, 4 \mathrm{n}\right)$ reaction at $162 \mathrm{MeV}^{9}$ is presented in Fig. 1. This band has also been observed in an

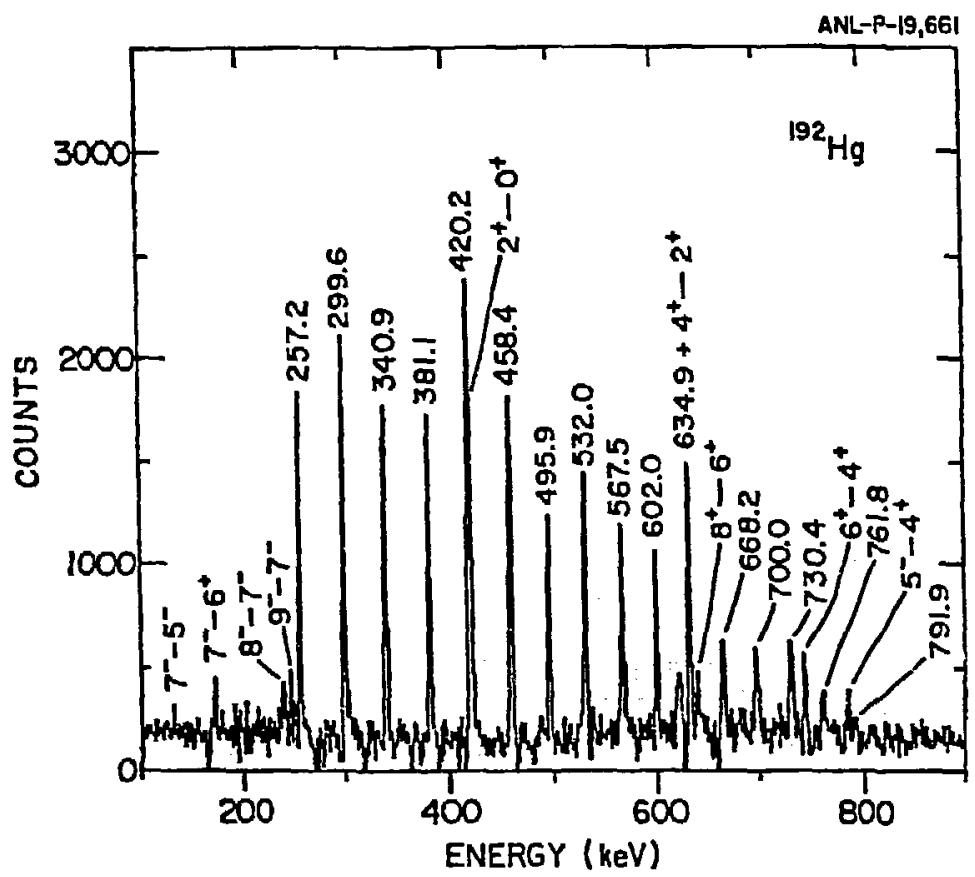

Fig. 1. Superdeformed band in ${ }^{192} \mathrm{Hg}$ (from Ref. 9.).

independent experiment by Becker et al.10, and the results are in good agreement. A band of 16 transitions extending in energy from 257 to $792 \mathrm{keV}$ was observed (an additional weak 215-keV transition is reported in Ref. 10 for which no conclusive evidence was found in our work ${ }^{9}$ ). The total flow through the band represents 1.97 of all transitions in ${ }^{192} \mathrm{Hg}$. From Fig. 1 it is clear that the band feeds the known levels up to $8^{+}$in the positive-parity yrast sequence and up to $9^{-}$in the negative parity band. Transitions which link the superdeformed band with known yrast levels could not be found. It is likely that many different decay paths share the intensity and that the link is statistical in nature. This assumption is supported by the observation that the feeding into the yrast states is spread over several states belonging to two bands of opposite parity. The avor-ge entry spin into the yrast states is \% 8 h. The spin of the lowest level in the SD band (fed by the 257-keV $\gamma$-ray) 
was estimated to be 10 from the the deexcitation pattern out of the superdeformed band, the average entry spin into the yrast states and the assumption of a $\Delta l=2 h$ angular momentum removal by the transitions linking the SD states and the yrast line ${ }^{9}$. The same spin vilue is also obtained in Ref. 10 from a procedure where $\mathrm{J}(2)$ is $f$ it by a power series expansion in $\omega^{2}$, which is then integrated to give the spin. The highest spin observed is then most probably 42K. With these assumed spin values, a static moment of inertia $J(1)$ can be derived: the latter is presented as a function of hw together with the values of $\mathrm{J}(2)$ in Fig. 2. It is striking that ( $I$ ) there is a large monotonic

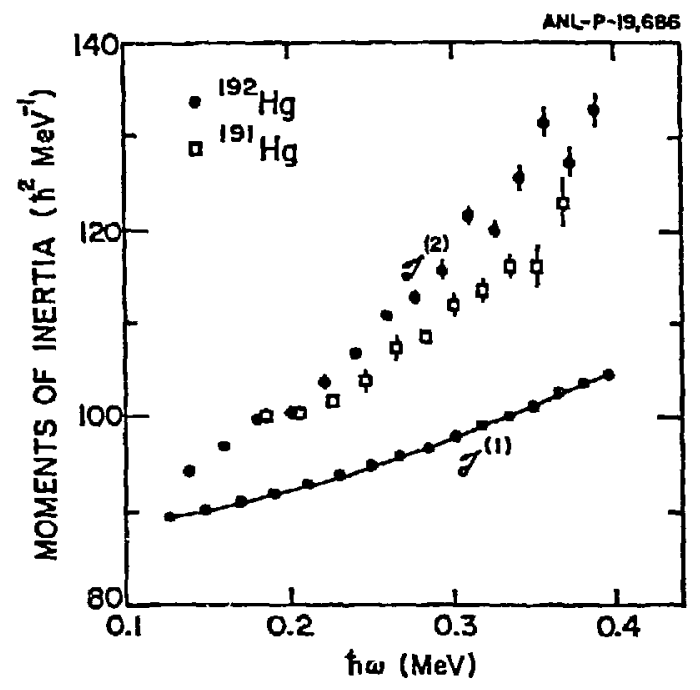

Fig. 2. Static and dynamic moments of inertia $J(1)$ and $J(2)$ for $192 \mathrm{Hg}$. $J(2)$ for ${ }^{191 \mathrm{Hg}}$ is given for comparison.

increase (40Z) in $J(2)$ with hw, (2) the $J(2)$ values for ${ }^{192} \mathrm{Hg}$ are similar, but always higher than those for ${ }^{191} \mathrm{Hg}$ at the same frequency (Fig. 2), and (3) $J(2)$ is significantly larger than $J(1)$ for all values of $h w$. All of these observations are a challenge for theory to explain.

Lifetimes within the SD band in ${ }^{192} \mathrm{Hg}$ were measured with the Doppler-shift attenuation method (DSAM) ${ }^{11}$. In contrast with previous measurements, where only fractional Doppler shifts $F(\tau)$ are reported 1,8 , we have been able to analyze detailed lineshapes for individual transitions between SD states $\left(\beta_{2} \sim 0.5\right)$. Analysis of individual lineshapes allows one to determine the variation of $Q_{t}$ as a function of $h_{w}$, as opposed to previous studies where $Q_{t}$ was assumed to be constant for all states in the band. In addition, information is also obtained on the feeding times into the SD structure. The measurement was performed with the reaction mentioned above at the beam energy of $159 \mathrm{MeV}$. The target was a $1 \mathrm{mg} / \mathrm{cm}^{2}$ isotopically enriched foil on which 14 
$\mathrm{mg} / \mathrm{cm}^{2}$ Au had been evaporated. An analysis in terms of broadened lineshapes was possible for transitions with $458 \leq E_{\gamma} \leq 700 \mathrm{keV}$. In order to extract lifetimes from these broadened lineshapes, the computer code LILIFI12 was used. Details of the analysis can be found in Ref. 11.

The lifetimes were transformed into transition quadrupole moments $Q_{t}(I)=\left(1.22<I 020 / I-2>T E \xi_{j}\right)^{-1 / 2}$ assuming the spin values given above. The $Q_{t}-$ values are displayed as a function of $\mathrm{t}_{\mathrm{w}}$ in Fig. 3b. As can be seen, the quadrupole moment $Q_{t}$, and hence the deformation, remain essentially constant over the entire frequency range. This result rules out centrifugal stretching as an explanation for the rise in $J^{(2)}$ : this is illustrated by the dashed Iine in Fig. 3b where the values of $Q_{t}$ have been derived assuming that the change in $J^{(2)}$ is entirely due to a variation in deformation. As already discussed by Ye et al. ${ }^{9}$, mean-field calculations without pairing, such as those by Chasman 5 or $\AA^{\circ} \operatorname{rg}^{13}$, give proton and neutron contributions to $\mathrm{J}(2)$ which remain essentially constant with $\not w \omega$. Thus, the increase in $J(2)$ cannot be accounted for by the occupation of specific high-j orbitals: this points to the need to examine the effects of pairing.

In order to interpret the resul $\mathrm{s}$ presented above, cranked shell model calculations with pairing were performed using a Woods-Saxon potential. The approach is similar to the one described in detail in Ref. 6,14 and 15 . In the discussion below, use is also made of the neutron and proton singleparticle routhian diagrams in Ref. 14. These calculations reveal a pronounced proton gap at $2=80$ which remains open for all frequencies considered. The resulting proton configuration contains fou $\mathrm{N}=6\left(i_{13 / 2}\right)$ protons $\left(\pi 6^{4}\right)$. For the neutron system, single-particle gaps exist at $N=112$ and $N=116,1 y$ ing between the second and the third $N=7\left(j_{15 / 2}\right)$ orbitals with $\Omega=3 / 2$ and $5 / 2$, respectively. The gaps are separated by the two deformation-aligned levels [512]5/2 and [624]9/2. Very similar single-particle level diagrams have been obtained in the Woods-Saxon model of Ref. 5. The resulting neutron configuration contains four $j_{15 / 2}$ neutrons and, thus, the SD configuration in $192 \mathrm{Hg}$ can be labelled $\left(\pi 6^{4} \nu 7^{4}\right)$. Figure 3 a compares the calculated dynamic moment of inertia with the data. In the calculation, pairing correlations were treated self-consistently by means of the particle number projection procedure6. The rise in the calculated $J(2)$ can be ascribed to the combined gradual alignment of a pair of $\mathrm{N}=6\left(i_{13 / 2}\right)$ protons and of a pair of $\mathrm{N}=7$ $\left(j_{15 / 2}\right)$ neutrons within the frequency range under consideration. The evolution of the nuclear shape with $h_{w}$ was also calculated 11 . The inset in Fig. 3b illustrates that within the frequency range of interest the predicted changes in $\beta_{2}$ and $\beta_{4}$ are very small. The resulting $Q_{t}$-values agree well with the measured values as is shown by the solid line in Fig. 3b. The success of 


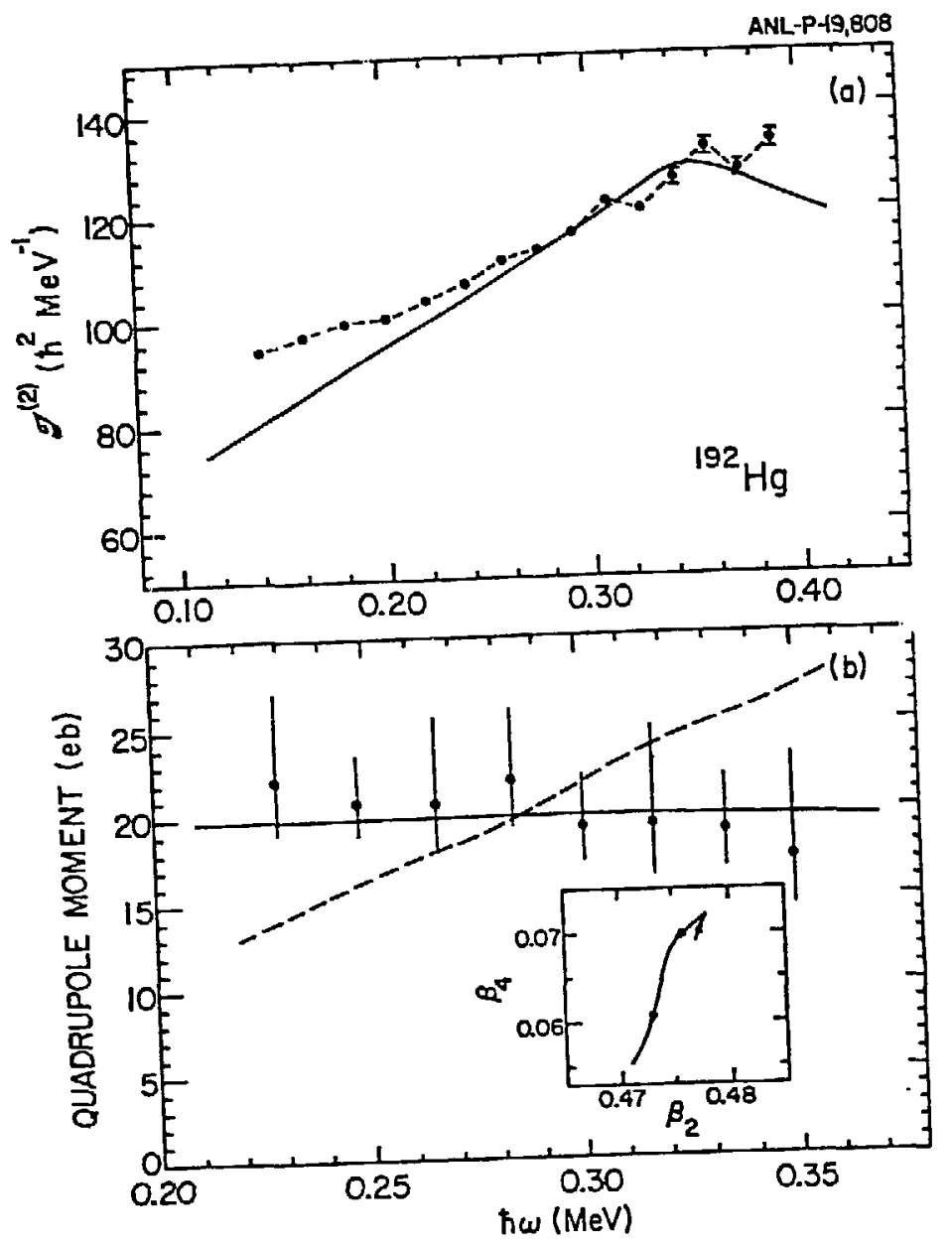

Fig. 3a) Comparison between the measured and calculated dynamic moment of inertia for ${ }^{192} \mathrm{Hg}$.

b) Comparison between the measured and calculated $Q_{t}$ values in the SD band of ${ }^{192} \mathrm{Hg}$. The dashed curve is a calculation assuming that the rise in $J^{(2)}$ is due to a change in deformation only, the solid line is a cranked shell model calculation. The inset shows the calculated change in deformation parameters $\beta_{2}$ and $\beta_{4}$ over the frequency range of interest.

the calculations in reproducing all aspects of the data allows us to propose that quasiparticle alignments and the resulting changes in pairing represent a major factor contributing to the rise in $\mathrm{J}(2)$ in $192_{\mathrm{Hg}}$ and, probably, in other nuclei in this region. Further evidence for this conclusion is discussed below.

As mentioned above, DSAM measurements also provide information on the sidefeeding lifetimes. If the sidefeeding is assumed to come from rotational bands with the same $J(2)$ as the SD band, then the resulting transition quadrupole moment for the sidefeeding Q $\mathrm{gf}$ can be derived in the analysis. The average values for $Q_{t}$ and $Q Q^{f}$ differ slightly, $20 \mathrm{eb}$ vs $16 \mathrm{eb}$. This difference probably reflects the proposed admixture 16 between SD levels and 
states of smaller deformation above the yrast line. However, the smaller $Q \mathrm{Q}^{\mathrm{f}}$ value could also be due, at least in part, to the approximations used in the description of the sidefeeding cascade in the analysis ${ }^{11}$.

3. NEUTRON AND PROTON EXCITATIONS: THE CASE OF ${ }^{191} \mathrm{Hg}$ AND ${ }^{193} \mathrm{Tl}$

We have shown in section 2 that the data for the "doubly magic" SD nucleus $192 \mathrm{Hg}$ can be described successfully in cranking calculations using a WoodsSaxon potential. The same calculations can also be tested in neighboring nuclei and specific predictions are available ${ }^{14}$. Detailed spectroscopy in the SD minimum has revealed multiple band structures in ${ }^{194} \mathrm{Hg},{ }^{193} \mathrm{Hg}$ and ${ }^{191} \mathrm{Hg}$ (Refs. 14, 15, 17-19). First, we shall compare experiment with calculations in ${ }^{191} \mathrm{Hg}$. Figure 4 presents spectra for the two new SD bands (bands 2 and 3 )

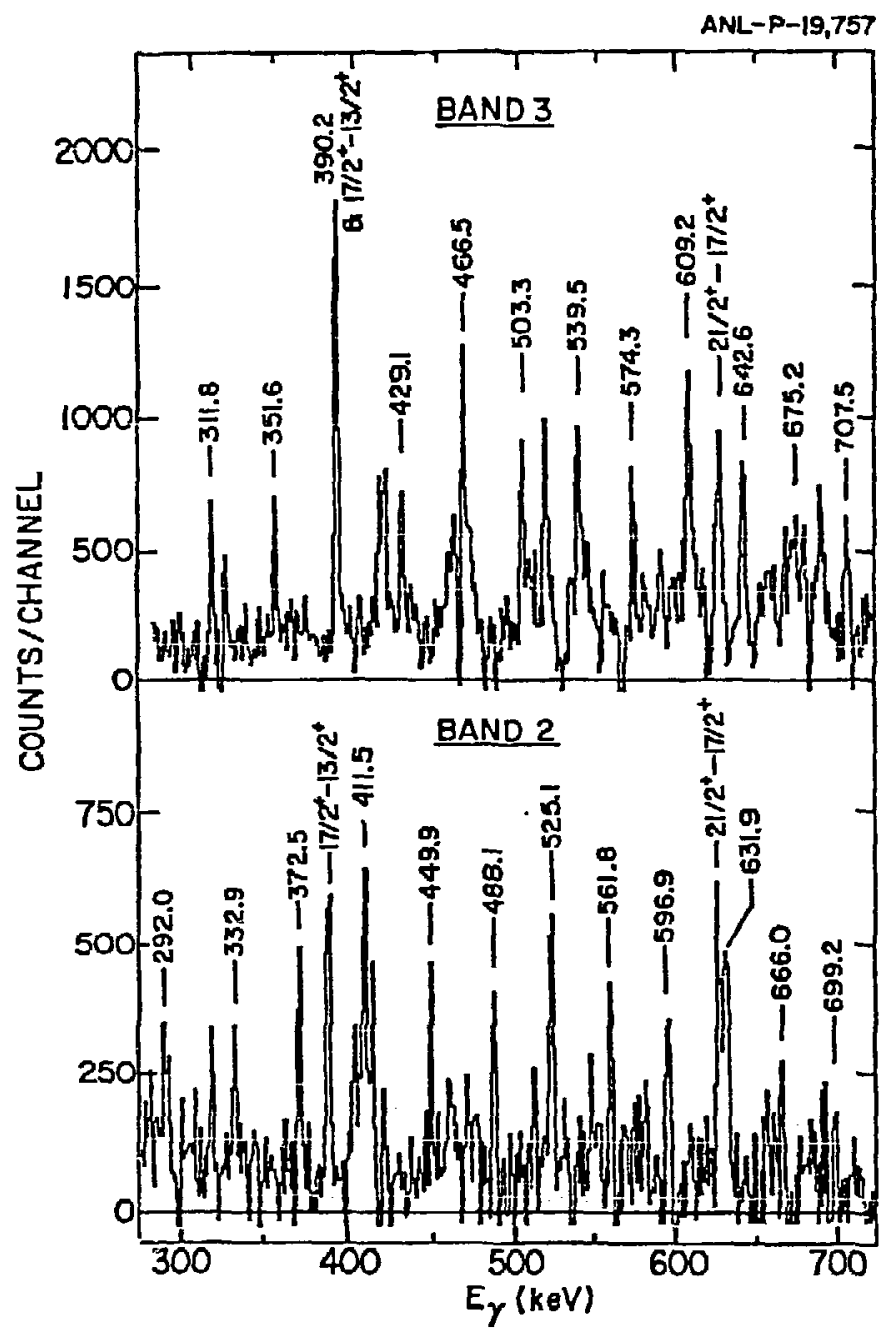

Fig. 4. Excited superdeformed bands in ${ }^{191_{\mathrm{Hg}}}$ (from Ref. 19).

in this nucleus. Each band corresponds to 0.87 of the events in ${ }^{191} \mathrm{Hg}$ (as measured with the ${ }^{160} \mathrm{Gd}\left({ }^{36} \mathrm{~S}, 5 \mathrm{n}\right.$ ) reaction at $172 \mathrm{MeV}$; the SD band reported earlier ${ }^{1}$ (band 1 ) has a corresponding intensity of 27). The three 
bands have essentially the same intensity pattern, i.e. the intensity remains constant in the frequency range $0.175<h \omega<0.24 \mathrm{MeV}$ and decreases gradually at higher rotational frequencies. The decay out of bands 2 and 3 occurs at lower frequency than in band 1 with the $\boldsymbol{\gamma}$-ray intensity decreasing gradually below $h_{w} w=0.175 \mathrm{MeV}$. Information on the lifetimes of the states was obtained for a few of the new transitions from a thick target experiment with the Dopplershift attenuation method (see Refs. 1, 15 for details): the deformation in the bands was found to be very similar.

Figure 5a presents the dynamic moments of inertia for the 3 bands as a function of hw. In all cases, $J(2)$ is again seen to increase with fiw. Furthermore, the average values of $J(2)$ for bands 2 and 3 (110 and $113 h^{2} \mathrm{MeV}^{-1}$, respectively) are somewhat larger than the corresponding value for band $1\left(108 \mathfrak{h}^{2} \mathrm{MeV}^{-1}\right)$ and are close to the value reported for ${ }^{192 \mathrm{Hg}}$ $\left(113 \mathrm{~K}^{2} \mathrm{MeV}^{-1}\right)$. For $\mathrm{T}$-ray energies below $400 \mathrm{keV}$, the transition energies of band 3 are almost exactly intermediate to the energies of band 2: the situation is similar to that reported ${ }^{14}$ for ${ }^{194} \mathrm{Hg}$ where the two excited bands exhibit the same feature over a larger energy range. In analogy with Ref. 14, bands 2 and 3 are interpreted as "signature partners". The degree of signature splitting can then be inferred from Fig. Sb where the experimental

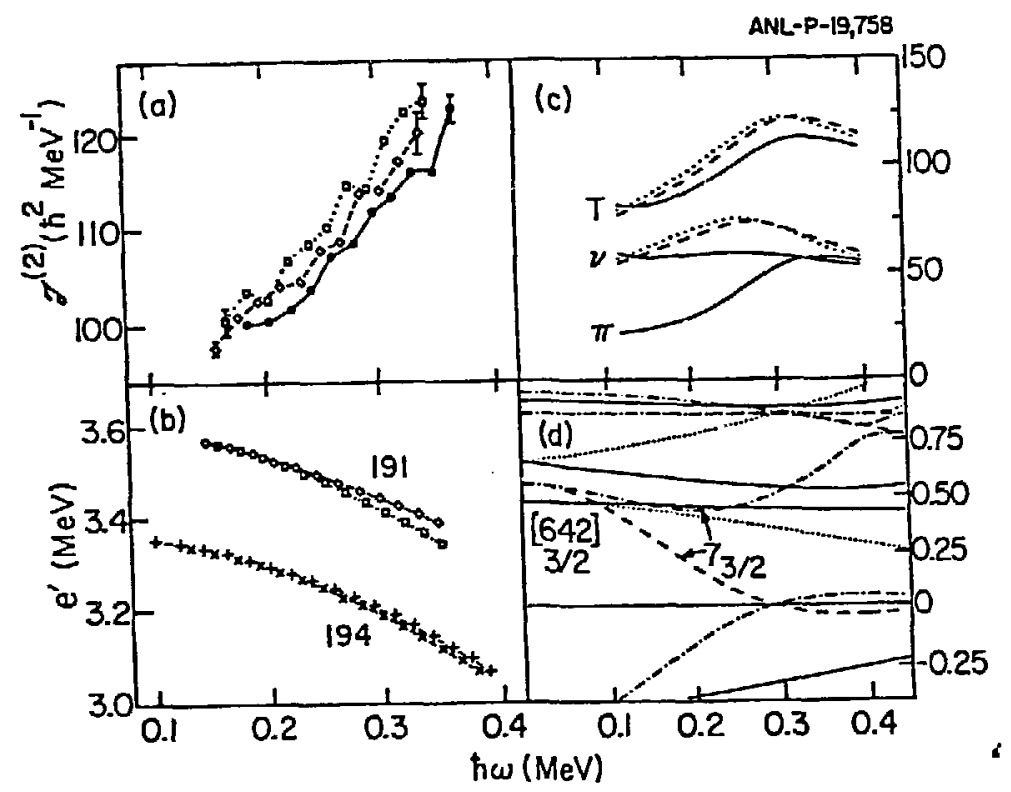

Fig. 5. Experimental (a) and calculated (c) dynamic moments of inertia for the three SD bands in $191_{\mathrm{Hg}}$. Experimental single-particle routhians for the excited SD bands in ${ }^{191} 1_{\mathrm{Hg}}$ and $194 \mathrm{Hg}$ (b) and quasiparticle routhians for $\mathrm{N}=111$ (d) (see Ref. 19 for details).

single-particle routhians $e^{\prime}$ for bands 2 and 3 are presented as a function of fiw. These routhians were calculated under the assumption that the bands are strongly coupled at low fiw. The excitation energies were arbitrarily taken as 
4.5 MeV and $4.64 \mathrm{MeV}$ for bands 2 and 3, respectively, and the spins were estimated with the method proposed in Ref. 10. We note that the spins derived in this way, i.e. $25 / 2$ and $27 / 2$ for the bandheads of bands 2 and 3 , respectively, differ by one unit and are consistent with the strong coupling picture presented here. The energy of the core was approximated by a Harris parametrization from fits to the low levels of the known yrast SD bands in

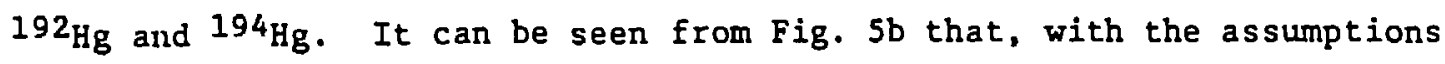
outlined above, the two bands may be proposed as signature partners for fiw<0.2 $\mathrm{MeV}$ (i.e. they follow the same smooth trajectory) and exhibit increasing signature splitting for hu>0.2 MeV. This behavior differs from that observed 14 in ${ }^{194} \mathrm{Hg}$ (shown for comparison in Fig. 5b): in this case the two excited bands show very little signature splitting over the entire frequency range.

The results can be compared with the cranked shell model calculations described above. Figure 5d presents the neutron quasiparticle routhians for $191_{\mathrm{Hg}}$. The yrast SD configuration is built on the aligned $\nu 7_{3} / 2$ routhian with parity and signature quantum numbers $(\pi=-, r=+i)$. We associate this configuration with band 1 . Bands 2 and 3 can be understood as the signature partners built on the [642]3/2 orbital with quantum numbers $(\pi=+, r=+i)$ and $(\pi=+, r=-i)$, respectively. The evolution of the calculated $J(2)$ values for the 3 bands as a function of $t_{w}$ is given in Fig. 5c where the individual

contributions of the proton and neutron configurations under discussion are also presented. The major contribution to the rise of $J(2)$ in the three bands is caused by the gradual alignment of the $\pi 6^{4}$ protons. The neutron contributions differ for band 1 and bands 2 and 3 . In band 1 , the $N=7$ neutron crossing is blocked whereas it is present in bands 2 and 3 . As a consequence, the neutron contribution to $J(2)$ remains almost constant for band 1 while an additional increase is calculated for bands 2 and 3 . The calculations reproduce the data rather well for $h w \geq 0.2 \mathrm{MeV}$ as can be seen from a comparison between Fig. $3 \mathrm{a}$ and $3 \mathrm{c}$. As discussed in Ref. 6, the larger deviations between theory and experiment at lower frequencies can be attributed to the renormalization procedure used in the calculations. The signature partner of band $1(\pi=-, r=-i)$ is calculated tc lie several hundred keV higher in excitation energy because of the large signature splitting (Fig. 5d) and, as is result, the corresponding rotational band should be more difficult to observe experimentally. The calculated values of $J(2)$ in bands 2 and 3 are similar to that of the lowest SD band in ${ }^{192} \mathrm{Hg}$ since all three have the same content in intruder orbitals (four $N=6\left(i_{13 / 2}\right)$ protons and four $N=7\left(j_{15 / 2}\right)$ neutrons). Furthermore, it is seen in Fig. 5d that the $\nu[642] 3 / 2$ routhians form a coupled structure below hw $\sim 0.15 \mathrm{MeV}$, while signature splitting increases gradually 
at higher frequencies. In the calculations without pairing, this signature splitting is present as well, although slightly reduced ${ }^{14}$. These features are present in the data and can be considered as strong arguments in favor of the assignments presented here. It should, however, be mentioned that another possible assignment for bands 2 and 3 could involve the neutron orbitals [512]5/2 and [624]9/2 mentioned above. This possibility cannot be excluded a priori as the calculations are expected to reproduce the positions of the single-particle states to within a few hundred keV only. However, these orbitals are calculated to remain strongly coupled over the entire range in thw and no significant signature splitting would result. This is contrary to the experimental observations and reinforces our assignment based on the $\nu[642] 3 / 2$ orbital for this SD nucleus.

An equally successful description in terms of the cranked shell model has been presented for ${ }^{194} \mathrm{Hg}$ in Ref. 14 . In this nucleus, 3 SD bands have been observed in separate experiments at Daresbury 14 and at Berkeley 17 . The lowest neutron excitations are predicted to involve transitions from the [512]5/2 to the [624]9/2 Nilsson level. This leads to pairs of strongly coupled bands showing no signature splitting. Bands 2 and 3 in this nucleus are interpreted as a signature partner pair since the $\gamma$-ray energies of band 3 are observed to lie mid-way between those of band 2 to within $1 \mathrm{keV}$ and both bands are of similar intensity. The bands observed 18,19 recently in $193^{\mathrm{Hg}}$ can be described in a similar way.

We have recently completed a study 20 of ${ }^{193} \mathrm{~T} 1$ which has one proton outside the $\mathrm{Z}=80, \mathrm{~N}=112$ core. This nucleus was produced under similar conditions (angular momentum input and excitation energy) as all the nuclai discussed above with the reaction ${ }^{160} \mathrm{Gd}\left({ }^{37} \mathrm{Cl}, 4 \mathrm{n}\right){ }^{193} \mathrm{~T} 1$ at $167 \mathrm{MeV}$. Two SD bands of 13 transitions each were found (see Fig. 6). The total $\boldsymbol{\gamma}$-ray flux through the two bands is about equal and corresponds at most to 0.57 (per band) of all decays in $193 \mathrm{~T} 1$. Because of the weak population, information on the decay towards the known yrast states is rather fragmentary and judged insufficient to propose spins on the basis of the observed feeding pattern into the yrast line. We note that the two bands persist to frequencies somewhat lower than those seen in $192 \mathrm{Hg}$ and comparable to those reported 14,17 for ${ }^{194} \mathrm{Hg}$. Figure 7 a presents the dynamic moments of inertia $J(2)$ for the two bands as a function of hw and also provides a comparison with $192 \mathrm{Hg}$. Again, J(2) increases with fw for both bands even though the rise occurs mainly for hw>0. $\mathrm{MeV}$ : the situation has some resemblance to that observed in band 1 of ${ }^{191} \mathrm{Hg}$ where $J(2)$ is also flat at the lowest frequencies. From Fig. 6, it can also be seen that for the two bands the transition energies are almost intermediat to one another, at least up to an energy of $400 \mathrm{keV}$. As in the case of 


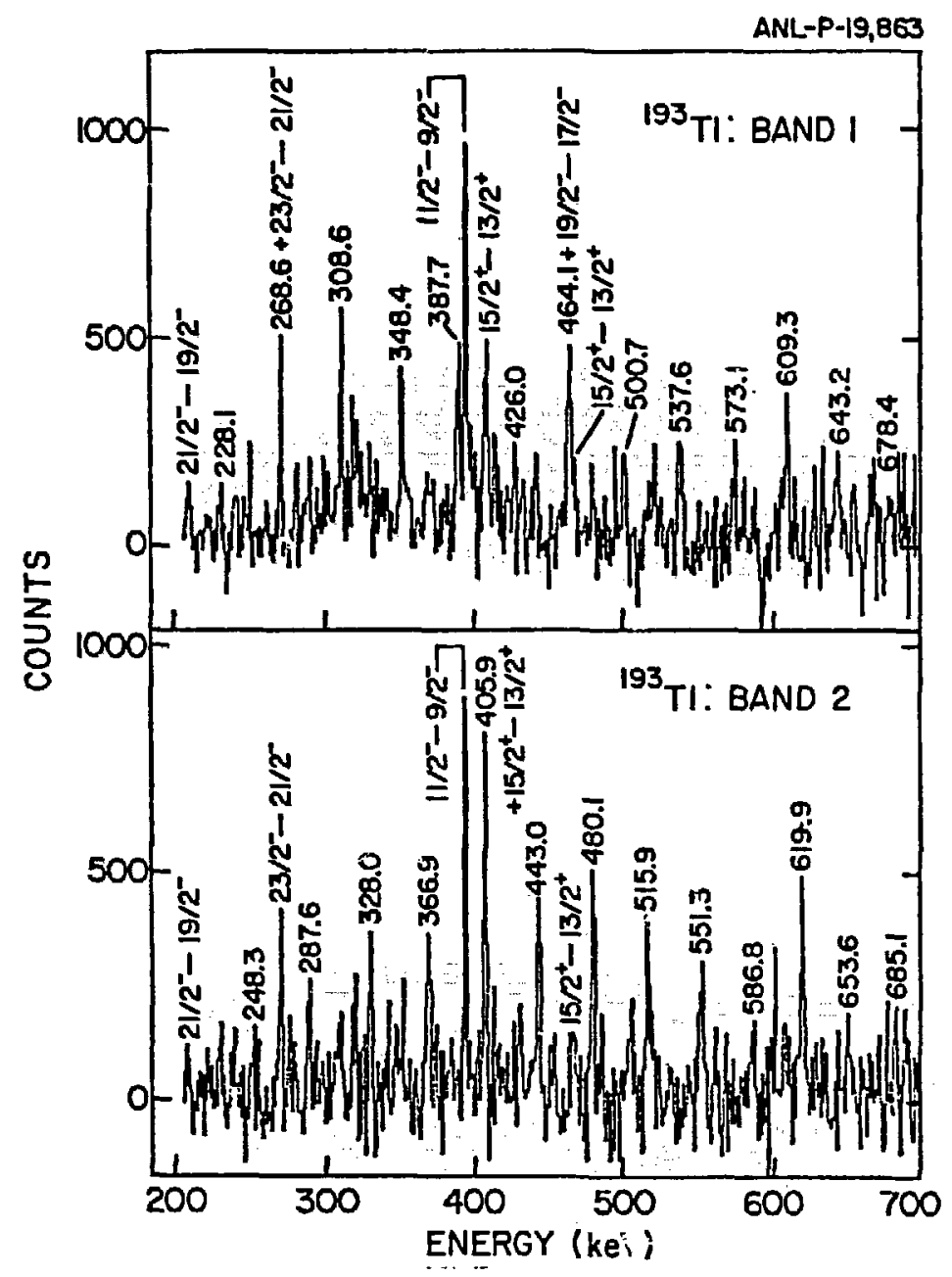

Fig. 5. Superdeformed bands in ${ }^{193} \mathrm{Tl}$ (from Ref. 20).
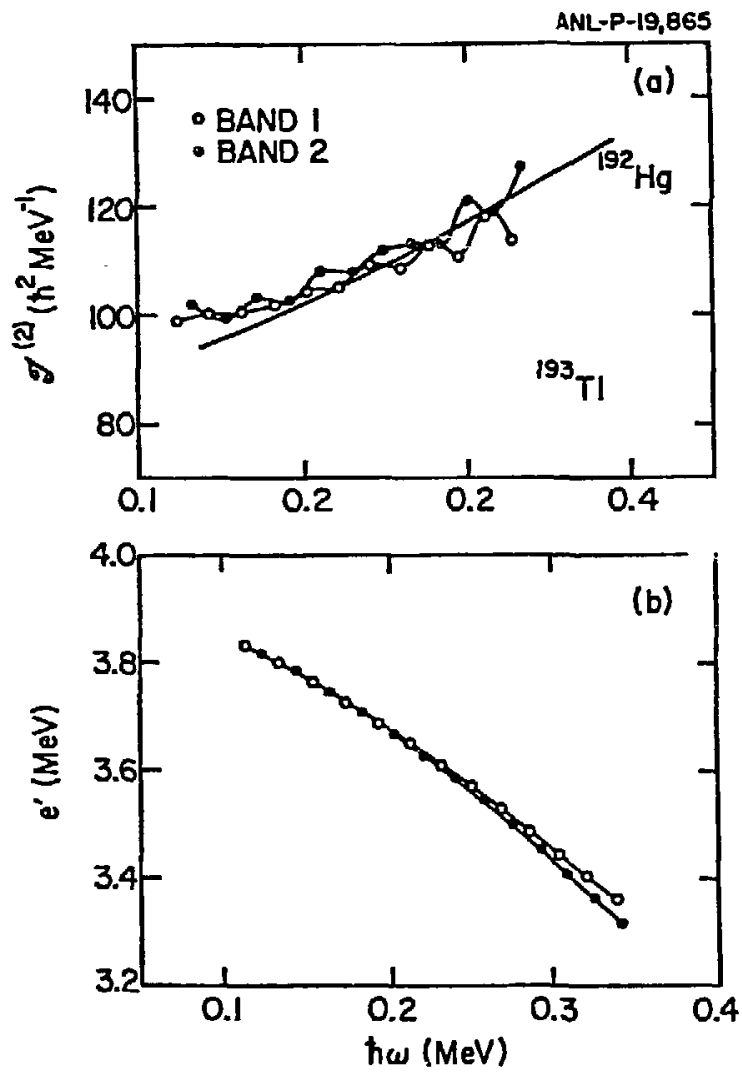

Fig. 7a) Dynamic moments of inertia for the SD bands in ${ }^{193} \mathrm{~T} 1$. ${ }^{192} \mathrm{Hg}$ is given for comparison.

b) Experimental routhians for the SD bands in ${ }^{193} \mathrm{Tl}$. 
bands 2 and 3 of ${ }^{191} \mathbf{H g}$, we present experimental single-particle routhians e' as a function of fut in Fig. 7b. The computation of these routhians was also performed assuming excitation energies of 4.5 and $4.61 \mathrm{MeV}$ for the bands 1 and 2, respectively. The spins were estimated with the method of Ref. 10 and found to be $19 / 2$ and $21 / 2$ at the bottom of the two bands. A Harris parametrization to the levels of the SD band in $192 \mathrm{Hg}$ was used to obtain the energy of the rotating core. All the evidence strongly suggests that the two bands can be considered as signature partners. At the lowest. frequencies the bands are strongly coupled while splitting increases gradually above hw=0.2 MeV.

The results can again be interpreted very nicely within the cranked shell model calculations with pairing. Above the $z=80$ gap 1 ies the third $i_{13 / 2}$

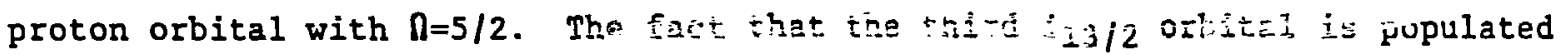
will result in an insrease in the value of $\mathrm{J}(2)$ with respect to $192 \mathrm{Hg}$ at the lowest frequencies as observed experimentally. At low frequency, the two signature partners for this orbital are degenerate and a strongly coupled structure is expected. Splitting is calculated to occur at frequencies $h_{w}>0.2$ $\mathrm{MeV}$, as seen experimentally.

From the discussion above it can be concluded that a good description of the SD bands can be achieved. Two points need, however, to be reemphasized: -(i) the inclusion of pairing is crucial for reproducing the data and, in particular, the smooth increase of $\mathrm{J}(2)$ with ${ }^{\prime} w$. The pairing correlations are calculated to be rather weak and are therefore mainly of dynamical character. In the proton system, pairing is reduced by the presence of the $z=80$ closure. As was shown in Refs. 9 and 14, the calculations require that the neutron pairing be reduced as vall if one wants to reproduce the similarities in the behavior of $\mathrm{J}(2)$ with tw observed in all nuclei ir: this region. -(ii) as pointed out in Ref. 21, the transition energies in some of the SD bands are very close to SD bands in neighboring nuclei (for example, transitions in bands 2 and 3 in ${ }^{191} \mathrm{Hg}$ are within 1 to $2 \mathrm{keV}$ of transitions in bands of $193 \mathrm{Hg}$ ) and all bands can be related to $192 \mathrm{Hg}$. These similarities have been linked to intrinsic spin alignments and underlying symmetries which are discussed by F. S. Stephens in the following presentation.

\section{LIMITS OF THE SUPERDEFORMED REGION FOR N-DEFICIENT Hg ISOTOPES}

First SD bands have also been found 22 in the nuclei ${ }^{190} \mathrm{Hg}$ and ${ }^{189} \mathrm{Hg}$. The SD band in ${ }^{190} \mathrm{Hg}$ (Fig. 8) was found in the ${ }^{160} \mathrm{Gd}\left({ }^{34} \mathrm{~S}, 4 \mathrm{n}\right.$ ) reaction at $160 \mathrm{MeV}$, i.e. under experimental conditions similar to those where the production of the SD bands in $191,192 \mathrm{Hg}$ was optimal23. A band of 12 transitions was found 


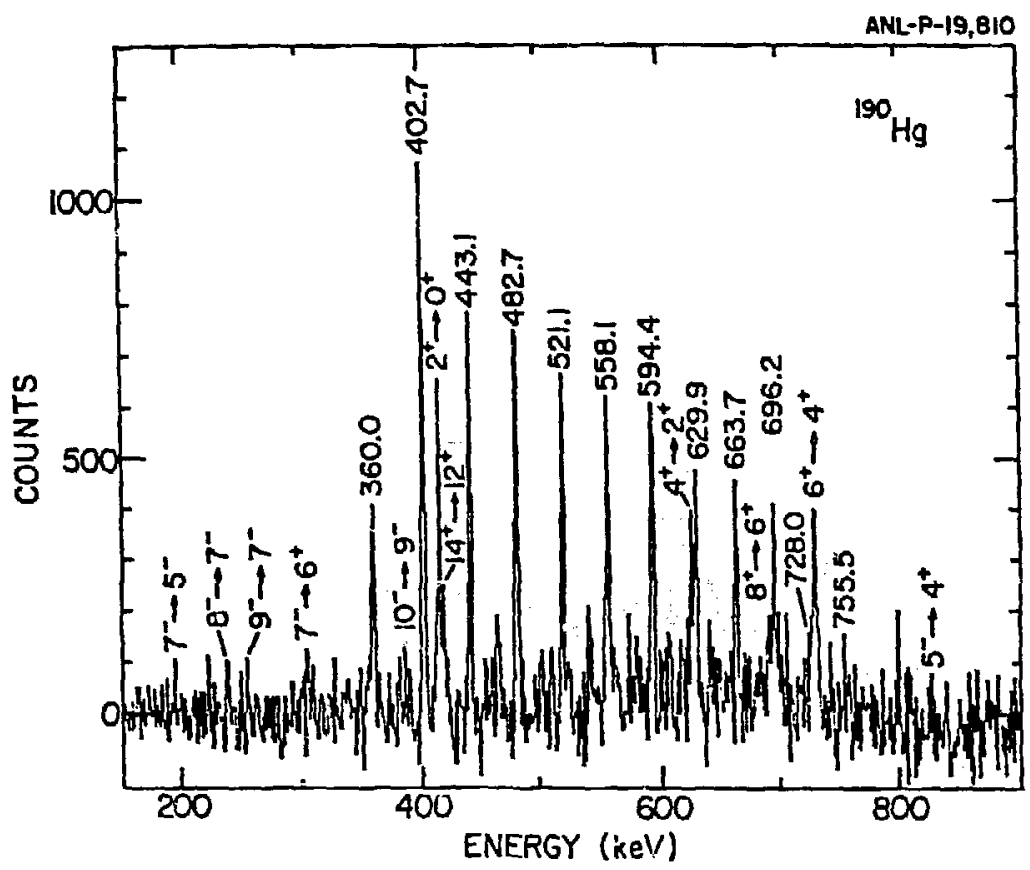

Fig. 8. Superdeformed band in $190_{\mathrm{Hg}}$ (from Ref. 22).

with a total intensity of only $0.8 z$ of the total $\gamma$-ray flow through the nucleus (the comparable number for the heavier $\mathrm{Hg}$ isotopes was $2 \pi$ ). The band - Iso decays towards the yrast line at higher frequency (the lowest observed transition has an energy of $360.0 \mathrm{keV}$ ) and, hence, most likely also at higher spin than is the case for ${ }^{192} \mathrm{Hg}$. (The fact that yrast states up to the $14^{+}$ state are observed in coincidence with the SD transitions confirm that the So band decays from states with higher spin tinan in e.g. ${ }^{192} \mathrm{Hg}$ ). An even weaker band ( $\leq 0.57$ of $\gamma$-flow) was found in ${ }^{189} \mathrm{Hg}$ with the $\left({ }^{34} \mathrm{~S}, 5 \mathrm{n}\right)$ reaction at 160 $\mathrm{MeV}$, a beam energy which may not be optimal for this reaction channel. In this case also the lowest observed transition has higher energy: $366.0 \mathrm{keV}$. For both nuclei, a rise in $J(2)$ with fiw is present but the absolute values of $J(2)$ at a given frequency are somewhat lower than for ${ }^{192} \mathrm{Hg}$. In terms of the discussion within the cranked shell model, this may reflect the fact that fewer neutrons are now occupying the $j_{15 / 2}$ intruder orbital.

The case of ${ }^{188} \mathrm{Hg}$ is potentially of great interest in that a rotational band structure built on a prolate shape of moderate deformation $(\beta \sim 0.2)$ is known to coexist at the lowest spins with the oblate yrast structure 24 and one may wonder whether a superdeformed minimum might be observabie as well. This nucleus was studied with the ${ }^{156} \mathrm{Gd}\left({ }^{36} \mathrm{~S}, 4 \mathrm{n}\right)$ reaction at $167 \mathrm{MeV}$, i.e. again under conditions of angular momentum input and excitation energy where the $S$.) bands in the heavier nuclei are populated optimally 23 . The coincidence data set contains in excess of $10^{8}$ events from which high multipli:-ity $\boldsymbol{\gamma}$-ray cascades in ${ }^{188} \mathrm{Hg}$ can be extracted with high efficiency through suitable total 
multiplicity and sum-energy gating. The spectra are of excellent quality. For example, the spectroscopy of the prolate band structure can be greatly extended over previous work 24 (states up to at least $26^{+}$have been identified), but to date no evidence for a SD band has been found. While the data analysis is still in progress, one can state that any SD band, if present, will have very small intensity $(\leq 0.5 z)$. From the findings above it is suggested that the limits of the SD region for the neutron deficient Hg isotopes may have been reached.

Figure $9 a$ presents as a function of mass the energy of the lowest transition in the SD bands of the $\mathrm{Hg}$ isotopes. In cases where several bands are known, the most intense band has been considered. A strong dependance with mass is obvious. Keeping in mind that gamma-ray energies and spins appear to be strongly related for these bands, Fig. 9a also implies that the SD minimum persists to lower spins as one remains closer to the $\mathrm{N}=112$ gap. The large difference in the spin at which the decay occurs contains substantive physics information since several factors affect the decay: the well-depth $W$ and the excitation energy $E^{*}$ of the superdeformed minimum; the level density of the normal states at $E^{*}$; and pairing, which has a large influence on the inertial mass parameter. Figure $9 \mathrm{~b}$ explores the dependence on $E^{*}$ and on the well-depth $W$. The curves are the results of cranked

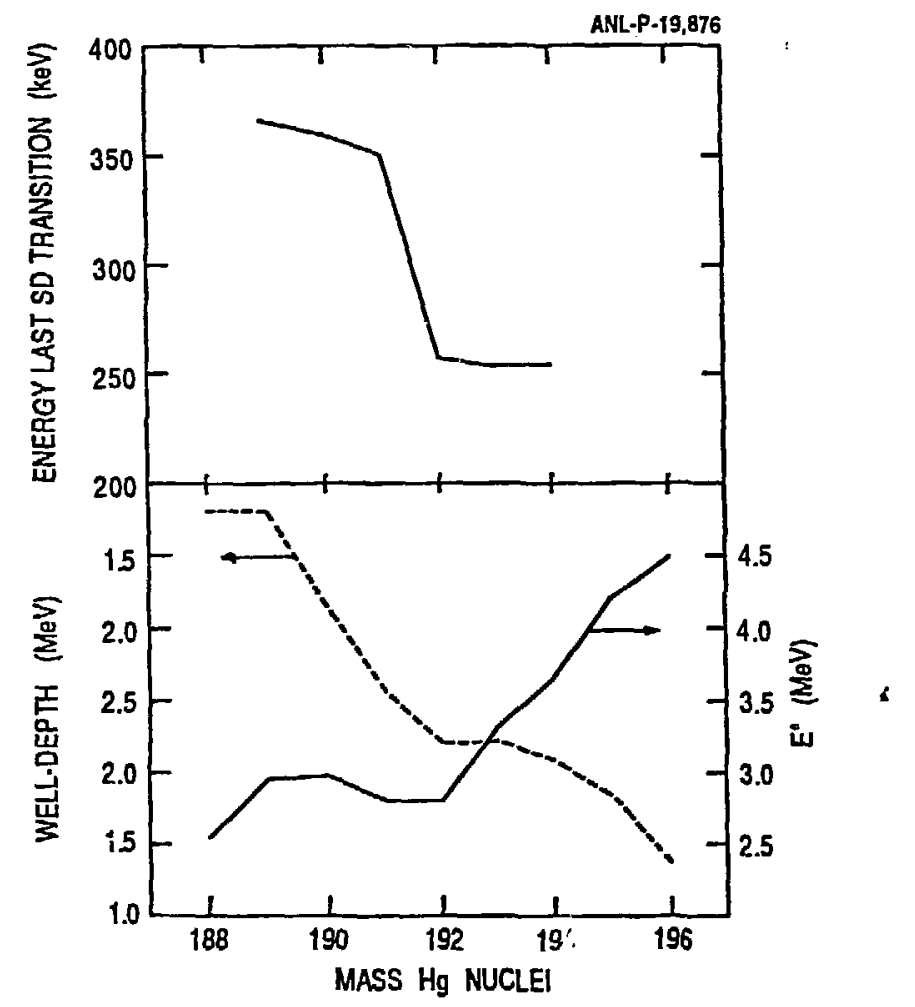

Fig. 9a) Energy of the lowest superdeformed transition in the yrast SD bands of the $\mathrm{Hg}$ isotopes.

b) Calculated well depth and excitation energy at spin 15 (Ref. 5). 
Strutinsky calculations by Chasman 5 and the values have been taken at the representative spin of $15 \mathrm{~h}$. In the calculations, the thickness of the barrier between the SD and the ground-state minima also increases with mass and the product between $W$ and the width of the barrier follows the same decreasing trend with increasing mass. This quantity is perhaps more relevant when deexcitation out of the SD minimum is considered. The data suggest that there is a strong correlation between the well-depth and the deexcitation out of the SD cascades, even though the role of $E^{*}$ should be given further consideration.

\section{CONCLUSIONS}

During the past year, considerable knowledge has been gained on superdeformation in the A 190 region. In fact, superdeformation is now known to occur in at least ten nuclei in this region and a total of 19 SD bands are known. The present experimental situation is summarized in Fig. 10 . From the

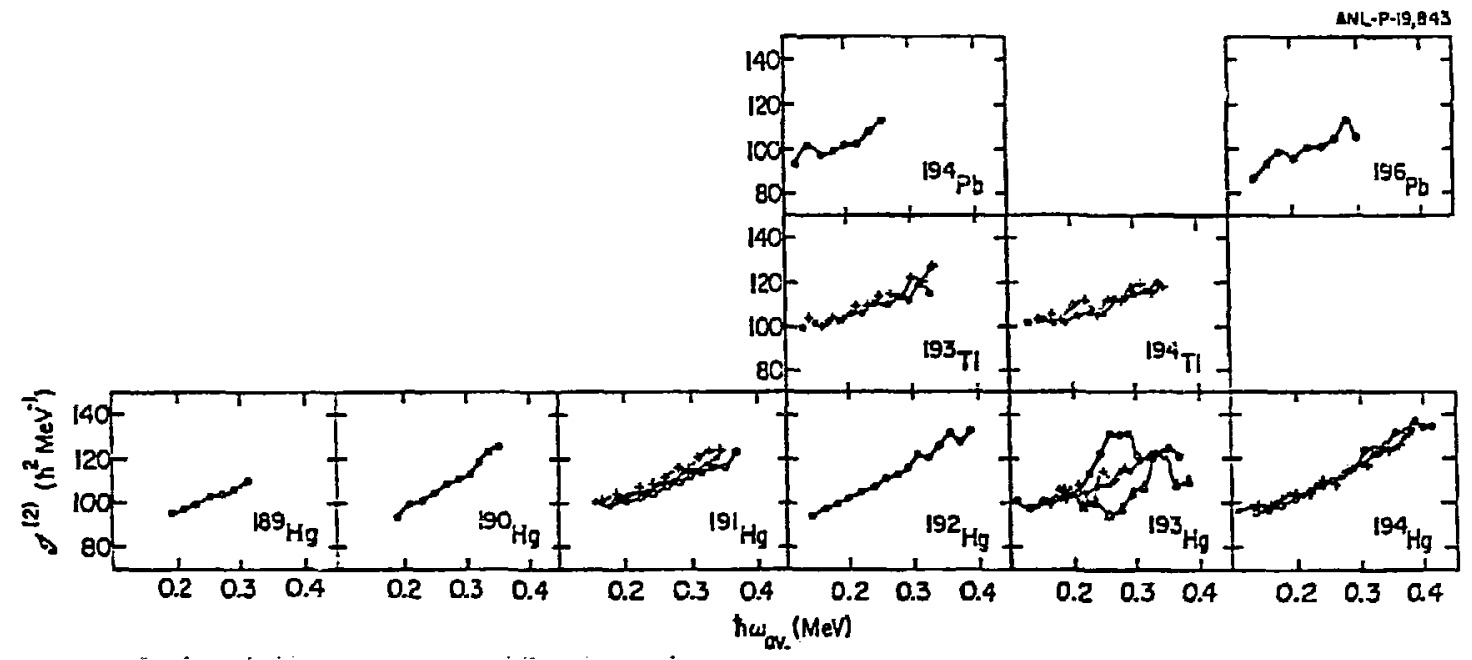

Fig. 10. Summary of the known SD bands in the $A \sim 190$ region. The data are from Ref. 1, 9, 10, 14, 15, 17-20, 22, 25 and 26 .

data discussed here, it was possible to rule out centrifugal stretching as the cause for the rise in $J(2)$ with hw. Consecutive alignment of $\nu j_{15 / 2}$ and $\pi i_{13 / 2}$ orbitals seems to provide a consistent explanation. The inclusion of pairing for reproducing the $J(2)$ behavior is crucial even though there is evidence that the pairing correlations are rather weak. These conclusions were obtained within the framework of the cranked shell model. It is, however, also realized that the model has its limitations. The following contribution by F. S. Stephens emphasizes the close correlation between the transition energies in many bands and those in the SD band of ${ }^{192} \mathrm{Hg}$ which strongly suggest the occurrence of intrinsic spin alignments reflecting an underlying symmetry in the SD nuclei. 
The authors express their deep gratitude to R. Chasman, W. Nazarewicz, M. Riley, F.S. Stephens and R. Wyss fur many stimulating discussions and S. Pilotte and F. Scarlassara for assistance in the ${ }^{193} \mathrm{Tl}$ experiment. This work was supported in part by the Department of Energy, Nuclear Physics Division, under contracts nos W-31-109-ENG-38, DE-AC07-76IDO1570 and DE-FG0287ER40346, by the National Science Foundation under grant PHY88-02279.

\section{REFERENCES}

1) E. F. Moore et al., Phys. Rev. Lett. 63 (1989) 360.

2) C. F. Tsang and S. G. Nilsson, Nucl. Phys. Al40 (1970) 275.

3) M. Caillian et al., Phys. Lett. 46B (1973) 11.

4) T. Bengtsson and I. Ragnarsson, Nucl. Phys. A436 (1985) 14.

5) R. R. Chasman, Phys. Lett. 219B (1989) 227.

6) W. Nazarewicz et al., Phys. Lett. 225B (1989) 208, and Nucl. Phys. A503 (1989) 285 .

7) T. Bengtsson, I. Ragnarsson and S. Åberg, Phys. Lett. 208B (1988) 39.

8) P. J. Nolan and P. J. Twin, Ann. Rev. Nucl. Part. Science 38 (1988) 533.

9) D. Ye et al., Phys. Rev. C41 (1990) R13.

10) J. Becker et al., Phys. Rev. C41 (1990) R9.

11) E. F. Moore et al., to be published.

12) H. Emling et al., Phys. Lett. 217B (1989) 33.

13) 5., Ảberg, Phys. Scr. 25 (1982) 23.

14) M. A. Riley et al., Nucl. Phys. A512 (1990) 178.

15) M. P. Carpenter et al., Phys. Lett. in print.

16) K. Schiffer et al., 2. Physik A332 (1989) 17.

17) C. W. Beausang et al., Z. Physik A335 (1990j 325.

18) E. A. Henry et al., Z. Physik A335 (1990) 361.

19) M. A. Riley, private communication and J. F. Sharpey-Schafer, contribution to this conference.

20) P. B. Ferrandez et al., to be published.

21) F. S. Stephens et al., to be published and F. S. Stephens, contribution to this conference.

22) M. W. Drigert et al, to be published.

23) T. L. Khoo et al, contribution to this conference and R. V. F. Janssens et al., to be published.

24) F. Hannachi et al., Nucl. Phys. A481 (1988) 135.

25) F. Azaiez et al., to be published.

26) K. Teine et al. and M. J. Brinkman et al., to be published. 